\title{
Copper-bearing Tourmaline Provenance from Chemometrics of LIBS and EMP Data
}

\author{
BARBARA L. DUTROW ${ }^{1}$, S. FARNSWORTH-PINKERTON ${ }^{1}$, \\ DARRELL J. HENRY ${ }^{1}$, NANCY J MCMILLAN. ${ }^{2}$ \\ ${ }^{1}$ Dept. Geology \& Geophysics, Louisiana State Univ., Baton \\ Rouge, LA, USA; dutrow@1su.edu \\ ${ }^{2}$ Dept. Geological Sciences, New Mexico State Univ., Las \\ Cruses, NM, USA; nmcmilla@nmsu.edu
}

Determining the provenance of gemstones is increasingly important in part, to: avoid 'conflict' gemstones which are subjected to embargos; ensure sustainable mining practices; and identify the country of origin which may have a substantial impact on pricing. Copper-bearing tourmalines (turs) can exhibit exquisite saturated 'neon' blue colors making them prized gemstones. Originally sourced from Batalha Mine, Paraíba, Brazil, they are now found in Nigeria and Mozambique. Their provenance has a substantial impact on gemstone price with Brazilian stones commanding a substantially higher price. Analytical and plotting methods for determining origin using trace elements are equivocal, typically resulting in overlapping fields of chemical data.

To evaluate alternative methods for provenance determination, 54 samples of Cu-bearing turs from two Brazillian and both African localities were analyzed using laser-induced breakdown spectroscopy (LIBS). Up to 64 spectra, each containing relative intensities of $\sim 24,000$ wavelenghts, were obtained and averaged to produce a single spectrum for each sample. These spectra were investigated using multivariate statistics (PCA, PLSR). Using a series of PLSR models clearly distinguishes the different localities. Models begin with those samples that cluster most tightly, Batalha. Once modeled, those samples are removed from the matrix. Other localities followed. The final grouping separated Mozambican and Nigerian samples. Samples were correctly identified with over $95 \%$ accuracy. One sample was consistently misidentified accounting for 5\% error; it had been cut with a saw and metal contaminant remained.

A subset of these samples were also analyzed for concentrations of 13 elements with EMP and subjected to the similar procedures using multivariate statistics. PLSR models clearly separate Brazilian and African samples with a high success rate of $>94 \%$.

While there are a number of caveats, results of this exploratory study demonstrate the potential of combining chemical data with multivariate statistics to develop a more powerful fingerprinting technique for discriminating $\mathrm{Cu}$ bearing tourmaline localities. 